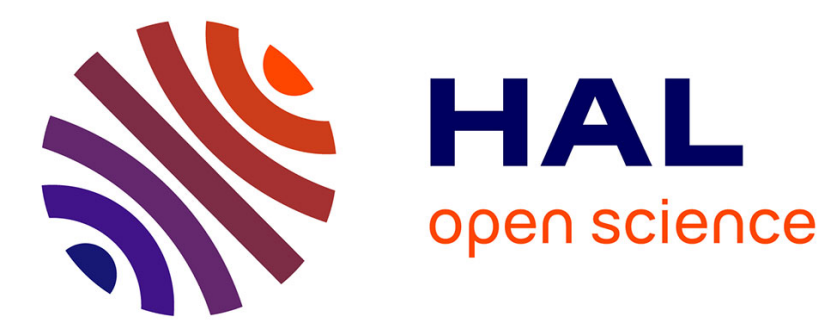

\title{
Hyperspectral Image Classification Using Tensor CP Decomposition
}

\author{
Mohamad Jouni, Mauro Dalla Mura, Pierre Comon
}

\section{To cite this version:}

Mohamad Jouni, Mauro Dalla Mura, Pierre Comon. Hyperspectral Image Classification Using Tensor CP Decomposition. IGARSS 2019 - IEEE International Geoscience and Remote Sensing Symposium, Jul 2019, Yokohama, Japan. 10.1109/IGARSS.2019.8898346 . hal-01998220

\section{HAL Id: hal-01998220 https://hal.science/hal-01998220}

Submitted on 14 Feb 2019

HAL is a multi-disciplinary open access archive for the deposit and dissemination of scientific research documents, whether they are published or not. The documents may come from teaching and research institutions in France or abroad, or from public or private research centers.
L'archive ouverte pluridisciplinaire $\mathbf{H A L}$, est destinée au dépôt et à la diffusion de documents scientifiques de niveau recherche, publiés ou non, émanant des établissements d'enseignement et de recherche français ou étrangers, des laboratoires publics ou privés. 


\title{
HYPERSPECTRAL IMAGE CLASSIFICATION USING TENSOR CP DECOMPOSITION
}

\author{
Mohamad Jouni, Mauro Dalla Mura, Pierre Comon \\ Univ. Grenoble Alpes, CNRS \\ Grenoble INP, Gipsa-Lab, 38000 Grenoble, France \\ firstname.lastname@gipsa-lab.fr http://www.gipsa-lab.grenoble-inp.fr
}

\begin{abstract}
Image classification has been at the core of remote sensing applications. Optical remote sensing imaging systems naturally acquire images with spectral features corresponding to pixels. Spectral classification ignores the spatial distribution of the data which is becoming more relevant with the development of spatial resolution sensors, and many works aim to incorporate spatial features based on neighborhood through for example, Mathematical Morphology (MM). Additionally, one could stack multiple morphological transformations of the image resulting in a highly complex block of data. Since classification is a tool that requires a matrix of samples and features, and simply stacking the different sets of features can lead to the problem of high dimensionality, we propose a way to create a matrix of low dimensional feature space by modeling the data as tensors and thanks to Canonical Polyadic (CP) decomposition. Experiments on real image show the effectiveness of the proposed method.
\end{abstract}

Index Terms - Remote Sensing Image, Mathematical Morphology, Attribute Profiles, Tensor Decomposition, Scene Classification.

\section{INTRODUCTION}

Classification of pixels has become a core subject in remote sensing applications allowing each pixel to be associated to a semantic label. For this procedure to take place in a pixelwise approach, it is necessary to have a set of samples and features, the former representing the pixels of a scene and the latter representing distinctive characteristics acquired from the scene.

Thanks to optical remote sensing systems, spectral features of the scene can naturally be acquired so that each pixel is associated to a discrete spectral signature. When the spectral acquisition spans a very high number of narrow bands, such images are referred to as hyperspectral images (HSI), represented as data cubes of 3 rd-order.

With the improvements in spatial resolution of acquired images, it has become easier to identify relatively small objects and more relevant to include spatial information as features. In fact, many works such in $[1,2,3,4,5,6,7,8]$ aimed at incorporating spatial features based on neighborhood information in addition to spectral ones, towards what is referred to as spectral-spatial classification. One way to account to spatial information in classification is by extracting features obtained by filtering an image with operators based on Mathematical Morphology.

Creating a new mode based on spatial MM features normally yields a block of data that is of order four: i.e., two spatial dimensions (image rows and columns), one to spectral and one to spatial features. Furthermore, one might want to stack multiple types of spatial transformations based on different concepts (e.g., size and shape) in a fifth mode, making it a data block of order five. As such, merging the first two modes that account to positioning will still give an array that is at least of order three, so in terms of classification, one way to deal with it is to reshape the dataset into a matrix, but this can still suffer from high dimensionality when the size of the spatial features is large, also this technique ignores the multimodal structure of the data and merges them together as one.

One natural approach to deal with the multi-way structure of the data is by using tensor analysis [9]. Tensors are data structures in multi-linear algebra, they are normally the extension of vectors and matrices into higher orders. Tensors are represented by multi-dimensional (or multi-way) arrays, hence they are a natural selection and a direct way to handle the problem of high-order features and account to the interactions between the different modes.

In short, we aim to model high-order-feature hyperspectral data in the framework of tensors and exploit their tools for the sake of classification. In fact, tensor modeling of HSI for classification has been explored previously in [10] where Tensor-Principal Component Analysis (TPCA), a highorder extension of PCA, is used as a method to maintain the spectral-spatial multi-modal structure and reduce the dimensionality of the data.

In this paper, we build a high-order tensor by stacking multiple types of spectral and spatial features separated in different modes, and we choose to jointly handle these features by taking advantage of the $\mathrm{CP}$ decomposition. We show through experiments on real image that $\mathrm{CP}$ decomposition is a good way to represent the pixel data directly in a "compact matrix form" and thanks to a low dimensionality of the de- 


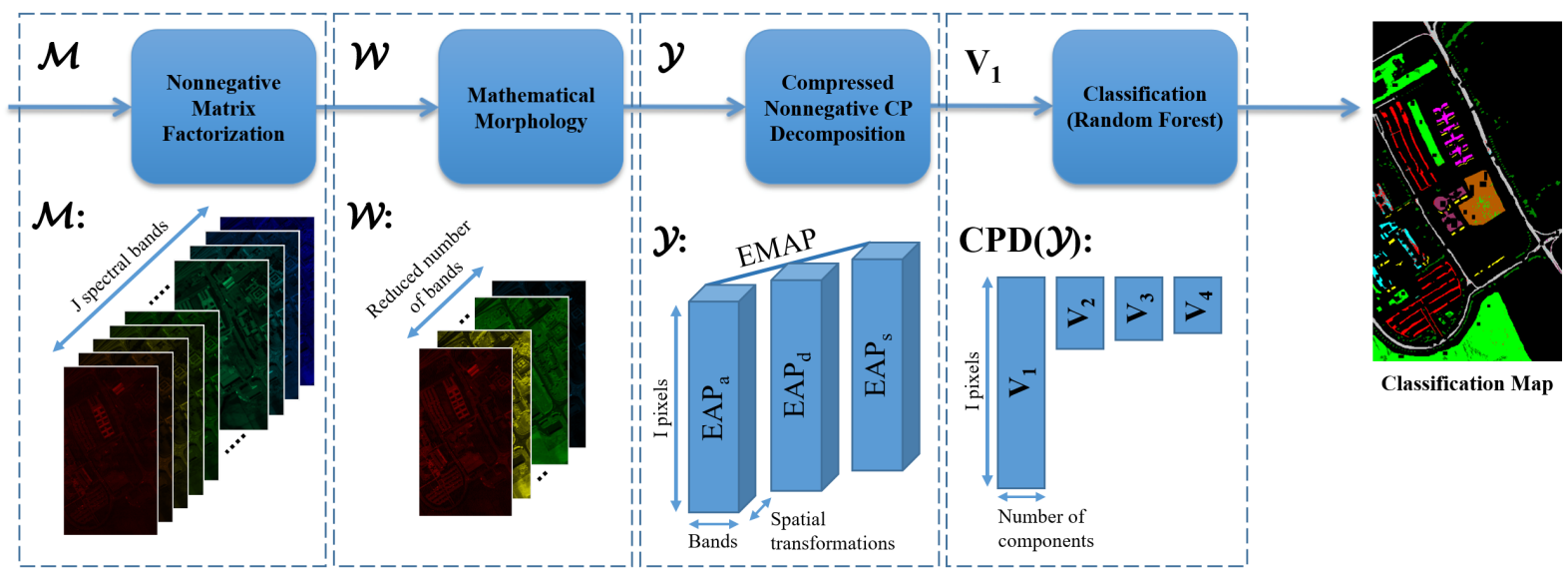

Fig. 1: Flow chart of the proposed method.

composition, leading to a low dimensionality of the feature space despite the high complexity of the data. Moreover, it is possible to incorporate nonnegative constraints into the $\mathrm{CP}$ decomposition, which allows to spectral unmixing in the future. Our approach to model multiple types of spatial features is inspired by the work of [4] in Extended Multi-Attribute Profiles (EMAP), where multiple types of morphological attribute operators are considered in the classification. In [4], the different Extended Attribute profiles (EAP) are concatenated along the third mode of the HSI. Instead, we keep the different EAP separated by stacking them as a new mode in the tensor. The proposed method incorporating multiple types of features shows promising results in terms of accuracy of classification compared to other methods in the literature.

From here on the paper is organized as follows. Section 2 introduces the proposed method and the challenges within. Section 3 shows the experiments that were carried on. Finally, a conclusion is drawn in Section 4.

\section{THE PROPOSED METHOD}

In this section we briefly introduce the main aspects of the proposed method, which is shown in the flowchart of Figure 1. There are originally two main steps before entering classification, the first one is creating the spatial features based on MM and the second one is the CP decomposition. However, due to challenges induced by high dimensionality of the spectral bands and the high number of pixels, two sub-steps are needed. First, we reduce the number of bands with very minimal loss as a preprocessing step, which is usually done using PCA, but since we care about conserving the nonnegativity aspect of the data (for spectral unmixing which is out of the scope of this paper), we perform the reduction using Nonnegative Matrix Factorization (NMF). Second, before the CP decomposition step, we compress the first mode of the tensor in order to reduce its size, which helps a lot with computing the $\mathrm{CP}$ decomposition in an efficient way.

\subsection{Nonnegative Matrix Factorization Preprocessing}

First let us denote a HSI by $\mathcal{M} \in \mathbb{R}^{I_{1} \times I_{2} \times J}$, where $I=I_{1} I_{2}$ is the number of pixels and $J$ is the number of spectral bands. We are interested in reducing the number of bands regardless of the pixel positioning, so $\mathcal{M}$ is reshaped in lexicographic order into $M \in \mathbb{R}^{I \times J}$ by merging the first two modes. NMF decomposes a matrix $M \in \mathbb{R}^{I \times J}$ into the product of two nonnegative matrices such that $\boldsymbol{M}=\boldsymbol{W} \boldsymbol{H}^{T}$, where $\boldsymbol{W} \in \mathbb{R}^{I \times R}$ and $\boldsymbol{H} \in \mathbb{R}^{J \times R}$, and $R$ is the number of components in the decomposition. Our interest is that $\boldsymbol{W}$ can be thought as a way to represent pixel data with $R$ spectral features while conserving nonnegativity for reasons mentioned earlier, where essentially $R<J$. $\boldsymbol{W}$ is then reshaped back to 3rd-order $\mathcal{W} \in \mathbb{R}^{I_{1} \times I_{2} \times J}$.

\subsection{Spatial Features Using Mathematical Morphology}

As a case example, the focus in this paper will be on Extended Attribute Profiles (EAP) [4] for their effectiveness in extracting distinctive spatial features. Nevertheless, the proposed approach can also extend to different approaches for incorporating spatial features.

The attribute profile (AP) of a 2D image with scalar values is formed by concatenating the attribute transformations obtained using successive values of the attribute parameter, the result is a 3rd-order data block. The EAP of a multivariate image is formed when the AP of the single 2D images are concatenated, the result is a 3rd-order data block, where the spectral and spatial features are stacked in one mode. Our take on EAP is a bit different, we prefer to separate the spectral and spatial modes such that the result of an EAP is a 4thorder block of data denoted as $\mathcal{T} \in \mathbb{R}^{I_{1} \times I_{2} \times J \times K}$, and $K$ is the number of attribute transformations.

Furthermore, we compute more than one type of EAP of $\mathcal{W}$. These EAP are then stacked in parallel to form a fifth mode which can be seen as an acquisition of different types of EAP. Finally, the fifth-order data block is reshaped by merg- 
ing the first two modes to form a fourth-order data block denoted by $\mathcal{Y} \in \mathbb{R}^{I \times J \times K \times L}$, which is ready to be dealt with using tensor decomposition, and $L$ denotes the number of EAP being computed.

\subsection{Compressed Tensor Decomposition}

Tensor $\mathcal{Y}$ can be seen as a high-order-feature hyperspectral data. Seeing as the first mode of $\mathcal{Y}$ is the one of pixels, i.e. samples, and seeing as $\mathcal{Y}$ is a fourth order tensor, this means that each pixel has a third-order tensor of features which is indeed complex and heavy to deal with for the sake of classification, and here comes the importance of tensor decomposition. Tensor decomposition is a tool used to break the high-order complex structure of tensors into multiple simpler structures of matrices, while accounting to the intrinsic interactions between the elements of the tensor.

One of the most important of such tools is the CP decomposition, which can be seen in two different ways. To begin with, an $N$ th-order tensor $\mathcal{D} \in \mathbb{R}^{I_{1} \times \cdots \times I_{N}}$ is referred to as decomposable if it can be written as the outer product of $N$ vectors $\boldsymbol{v}_{n} \in \mathbb{R}^{I_{n}}$ s.t. $n \in\{1 \ldots N\}: \mathcal{D} \stackrel{\text { def }}{=} \boldsymbol{v}_{1} \otimes \ldots \otimes \boldsymbol{v}_{N}$. On one hand $\mathrm{CP}$ decomposition allows to decompose a tensor into the sum of rank-1 decomposable tensors:

$$
\mathcal{Y}=\sum_{r=1}^{R} \lambda_{r} \mathcal{D}_{r}
$$

where the tensor rank, denoted by $R$, is the least number of terms such that the CP decomposition is exact. On another hand, it allows to decompose an $N$-th order tensor into $N$ matrices, usually referred to as "factor matrices" which are formed by stacking each set of $R$ vectors as columns, and a diagonal core tensor $\Lambda$, formed by filling the diagonal with values of $\lambda_{r}$, under the following notation:

$$
\mathcal{Y}=\left(\boldsymbol{V}_{1}, \ldots, \boldsymbol{V}_{N}\right) \cdot \boldsymbol{\Lambda}
$$

$\mathrm{CP}$ decomposition can adopt the nonnegativity constraints in its computation in order to yield results that can be physically interpretable, and this will be more relevant for future works.

Hence, one aspect that appears in computing the CP decomposition is that each factor matrix $\boldsymbol{V}_{n}$ is directly related to the $n$-th mode of $\mathcal{Y}$. In our case of application, we are interested that $\boldsymbol{V}_{1}$ directly describes the first mode of pixels by means of $R$ components which could be seen as a compact form of features, and one could say that the complex structure of pixels in $\mathcal{Y}$ boils down to row vectors of $R$ elements, and due to redundancies that are present in $\mathcal{Y}, R$ could be relatively of a small value that is acceptable when feeding $\boldsymbol{V}_{1}$ to the classifier.

It is worth noting here that since the number of pixels is huge compared to the other dimensions, a compressed nonnegative CP decomposition inspired by [11] and adopted by the algorithm Alternating Optimization - Alternating Direction Method of Multipliers (AO-ADMM) [12] is carried out.

\section{EXPERIMENTS AND RESULTS}

\subsection{Description of the dataset}

Our dataset is described by a real hyperspectral image, University of Pavia. It is an image acquired by the ROSIS sensor with geometric resolution of 1.3 meters. The image has $610 \times 340$ pixels and 103 spectral bands. The dataset contains a groundtruth image that consists of 9 classes: trees, asphalt, bitumen, gravel, metal sheets, shadows, self-blocking bricks, meadows, and bare soil. There are also 42776 labeled pixels available as test set and 3921 pixels available as training set, the latter is fixed at that.

\subsection{Experimental set-up}

$N M F$. We start with the HSI such that $\mathcal{M} \in \mathbb{R}^{610 \times 340 \times 103}$ and the number of pixels is $610 \times 340=207400$. As a preprocessing step in order to reduce the number of 103 bands, we find that the NMF with 30 components yields a reconstruction error of $0.89 \%$ where $\mathcal{W}_{30} \in \mathbb{R}^{610 \times 340 \times 30}$, and that with 40 components yields a reconstruction error of $0.65 \%$ where $\mathcal{W}_{40} \in \mathbb{R}^{610 \times 340 \times 40}$. We use both datasets for the following steps.

EAP features. We use three different types of EAP as mentioned in [4], the area of the regions $\left(\mathrm{EAP}_{a}\right)$ with values of $\lambda_{a}=\{100,500,1000,5000\}$, the diagonal of the box bounding the region $\left(\mathrm{EAP}_{d}\right)$ with values of $\lambda_{d}=$ $\{10,25,50,100\}$, and the standard deviation of the grey-level values of the pixels in the regions $\left(\mathrm{EAP}_{s}\right)$ with values of $\lambda_{s}=\{20,30,40,50\}$, bringing a wide diversity of features to the image. Having 4 values of $\lambda$ corresponds to $K=9$.

First we create the 3 EAP for each of $\mathcal{W}_{30}$ and $\mathcal{W}_{40}$ corresponding to $L=3$, then we stack them in a 5 th order tensor to which the outputs are $\mathcal{Y}_{30} \in \mathbb{R}^{207400 \times 30 \times 9 \times 3}$ and $\mathcal{Y}_{40} \in \mathbb{R}^{207400 \times 40 \times 9 \times 3}$ respectively. Denote by Extended Multi-Attribute Profile (EMAP) as the stacking of multiple types of EAP.

Compressed nonnegative CP decomposition. Now that we have the 4th-order tensor, we pass it to the $\mathrm{CP}$ decomposition step. As mentioned earlier, this part is adopted by the algorithm AO-ADMM with constraints of nonnegativity on all the modes in addition to constraint of compression only on the first mode. The factor matrices are randomly initialized using the absolute value of i.i.d. standard Gaussian distribution, and only the first factor matrix is compressed after that.

Classification. For all the classifications, we use a Random Forest (RF) classifier with the number of trees set to 100. Table 1 shows some results of various conducted tests, where we compare the proposed method to others in the literature, namely PCA as carried out in [4] for EMAP and TPCA as carried out in [10]. The comparisons are based on the Overall Accuracy (OA) and the Average Accuracy (AA) of the classification. For all the conducted tests, the same set of attribute parameters was chosen. 


\begin{tabular}{|c|c|c|c|}
\hline Method & Features & $\mathrm{OA}$ & AA \\
\hline PCA & 4 & 68.34 & 78.01 \\
\hline $\mathrm{NMF}_{30}$ & 30 & 73.79 & 85.10 \\
\hline $\mathrm{NMF}_{40}$ & 40 & 77.74 & 86.33 \\
\hline PCA + EMAP [4] & 108 & 91.12 & 90.36 \\
\hline EMAP + TPCA [10] & 100 & 90.60 & 91.65 \\
\hline $\mathrm{NMF}_{30}+\mathrm{EMAP}+\mathrm{CPD}_{30}$ & 30 & 93.96 & 92.50 \\
\hline $\mathrm{NMF}_{40}+\mathrm{EMAP}+\mathrm{CPD}_{30}$ & 30 & 94.20 & 95.85 \\
\hline $\mathrm{NMF}_{40}+\mathrm{EMAP}_{+} \mathrm{CPD}_{40}$ & 40 & $\overline{94.76}$ & 94.38 \\
\hline
\end{tabular}

Table 1: Records of various tests in terms of Overall Accuracy (OA) and Average Accuracy (AA). The number of features for each test appears in the second columns. The best results are in bold and the second best are underlined.

\subsection{Results and discussion}

Looking at Table 1, we can see that the best results are achieved by computing the EMAP on the NMFs and applying CPD afterwards. The proposed method shows improved accuracy compared to the competing techniques, PCA and TPCA. On a second note, we see that classifying the components obtained by NMF yields better accuracy than classifying the components obtained by PCA. In addition to good accuracy, obtaining nonnegative features using NMF has an advantage of conserving nonnegativity and extracting physical meaning from the data compared to features obtained using PCA.

\section{CONCLUSION}

In this paper, creating multiple spatial diversities thanks to mathematical morphology was explored for the sake of spectral-spatial classification of HSI. The corresponding high-order data was handled using tensor decomposition by jointly accounting to spectral and spatial features. Some challenges like reducing the high dimensionality of the spectral bands while conserving the nonnegativity of the data was dealt with using NMF, which also showed some advantages over choosing PCA. Experimental results using a real HSI showed that the CP decomposition is a promising candidate to represent high-order feature data in low dimensional feature space with good accuracy. In future works, we plan to dig more into the properties of nonnegativity and investigate other potential interests of the CP decomposition, like spectral unmixing of HSI.

\section{REFERENCES}

[1] L. Bruzzone and L. Carlin, "A multilevel context-based system for classification of very high spatial resolution images," IEEE transactions on Geoscience and Remote Sensing, vol. 44, no. 9, pp. 2587-2600, 2006.
[2] J.M. Duarte-Carvajalino, G. Sapiro, M. Vélez-Reyes, and P.E. Castillo, "Multiscale representation and segmentation of hyperspectral imagery using geometric partial differential equations and algebraic multigrid methods," IEEE Transactions on Geoscience and Remote Sensing, vol. 46, no. 8, pp. 2418-2434, 2008.

[3] J. Li, J.M. Bioucas-Dias, and A. Plaza, "Spectralspatial hyperspectral image segmentation using subspace multinomial logistic regression and markov random fields," IEEE Transactions on Geoscience and Remote Sensing, vol. 50, no. 3, pp. 809-823, 2012.

[4] M. Dalla Mura, J.A. Benediktsson, B. Waske, and L. Bruzzone, "Morphological attribute profiles for the analysis of very high resolution images," IEEE Transactions on Geoscience and Remote Sensing, vol. 48, no. 10, pp. 3747-3762, 2010.

[5] M. Fauvel, J. Chanussot, and J.A. Benediktsson, "A spatial-spectral kernel-based approach for the classification of remote-sensing images," Pattern Recognition, vol. 45, no. 1, pp. 381-392, 2012.

[6] M. Fauvel, Y. Tarabalka, J.A. Benediktsson, J. Chanussot, and J.C. Tilton, "Advances in spectral-spatial classification of hyperspectral images," Proceedings of the IEEE, vol. 101, no. 3, pp. 652-675, 2013.

[7] P. Ghamisi, M. Dalla Mura, and J.A. Benediktsson, "A survey on spectral-spatial classification techniques based on attribute profiles," IEEE Transactions on Geoscience and Remote Sensing, vol. 53, no. 5, pp. 23352353, 2015.

[8] P. Ghamisi, E. Maggiori, S. Li, R. Souza, Y. Tarabalka, G. Moser, A. De Giorgi, L. Fang, Y. Chen, M. Chi, et al., "Frontiers in spectral-spatial classification of hyperspectral images," IEEE Geoscience and Remote Sensing Magazine, 2018.

[9] P. Comon, "Tensors: a brief introduction," IEEE Sig. Proc. Magazine, vol. 31, no. 3, pp. 44-53, May 2014, hal-00923279.

[10] S. Velasco-Forero and J. Angulo, "Classification of hyperspectral images by tensor modeling and additive morphological decomposition," Pattern Recognition, vol. 46, no. 2, pp. 566-577, 2013.

[11] J. Cohen, R.C. Farias, and P. Comon, "Fast decomposition of large nonnegative tensors," IEEE Signal Processing Letters, vol. 22, no. 7, pp. 862-866, 2015.

[12] K. Huang, N.D. Sidiropoulos, and A.P. Liavas, "A flexible and efficient algorithmic framework for constrained matrix and tensor factorization," IEEE Transactions on Signal Processing, vol. 64, no. 19, pp. 5052-5065, 2016. 